\title{
Gesamtinhaltsverzeichnis Klio Band 67, 1985
}

Wrlhelm Alzinger und Mitarbeiter, (Wien), Aigeira-Hyperesia und die Siedlung Phelloë in Achaia (I)

Gert Audring, (Berlin), Zur Arbeitswelt der Antike . . . . . . . . . . . . . 589

ILsE BECHER, (Leipzig), Augustus und der Kult der ägyptischen Götter . . . . 61

ILSE Becher, (Leipzig), Tiberüberschwemmungen. Die Interpretation von

Prodigien in Augusteischer Zeit . . . . . . . . . . . . . . . . . . .

Heinz Berthold, (Halle/S.), Das „klassische“ Zitat. Versus notissimi der Augu-

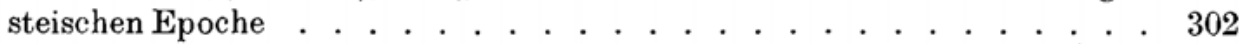

Jáxos Bollók, (Budapest), Die Figur des Mezentius in Vergils Aeneis . . . . . . 234

Stefan Borzsák, (Budapest), Zum neuen Horatius Teubnerianus . . . . . . 177

Gerda v. BüLow, (Berlin), Zu Problemen der römischen Keramik ～. . . . . . . 363

Gerda v. BüLow, (Berlin), Provinzialrömische Archäologie in Spanien . . . . $\quad 370$

Jan Burian, (Prag), Die Vergangenheit Roms im Rahmen der Augusteischen

Gegenwartspolitik . . . . . . . . . . . . . . . . . . . 29

Francis CaIrns (Liverpool), Concord in the Aeneid of Virgil . . . . . . . 210

Guatiero CaLboli, (Bologna), Wortstellung und literarische Nachahmung im vierten Odenbuch des Horaz

Maria Cytowska, (Warschau), Die Augusteischen Dichter in Polen im 16. Jahr-

hundert . . . . . . . . . . . . . . . . . . . . . . . 291

JÜRgen DeInINGER, (Hamburg), Livius und der Prinzipat . . . . . . . . . . . . 265

Hans-Joachim Diesner, (Halle/S.), Augustus und sein Tatenbericht. Die Res gestae Divi Augusti in der Vorstellungswelt ihrer und unserer Zeit . . . . . .

Trziano Dorand, (Neapel), Der, gute König' bei Philodem und die Rede des Maecenas vor Octavian (Cassius Dio LII, 14-40) . . . . . . . . . . . .

Dietrich Ebener, (Bergholz-Rehbrücke), Überlegungen aus Anlaß einer Vergil-Übertragung

Wolf-DIETER ERfURt, (Berlin), Augusteische Literatur aus verlegerischer Sicht.

Einige Anmerkungen zu den im Akademie-Verlag erschienenen zweisprachigen Ausgaben römischer Elegike

Thomas Fischer, (Bochum), Judäa im Hellenismus .......... . . 350

Bernd Funck, (Berlin), Das Bosporanische Reich aus der Sicht Strabons . . . 273

Hermann Funke, (Mannheim), Sunt lacrimae rerum. Komposition und Ideologie

in Vergils Aeneis . . . . . . . . . . . . . . . . . . . . . . . . .

IAMSE GAGUA, (Tbilissi), Die Sage von Hero und Leander bei Ovid . . . . . .

Rismag Gordestani, (Tbilissi), Zu den Prinzipien der kompositionellen Organisation in Ovids Metamorphosen . . . . . . . . . . . . . . . . . . . 198

RIgobert GÜNTHER, (Leipzig), Zum Landbesitz römischer Villen ． . . . . . . . 368

RIgobert GÜNther, (Leipzig), Römischer Staat und Kirche . . . . . . . . . . . 374 ASTRID HÄNDEL, (Rostock), Zur Interpretation von Inschriften mit Berufsbezeichnungen von Handwerkern und Händlern im Rom der Prinzipatszeit 
István HaHn †, (Budapest), Augustus und das politische Vermächtnis Caesars .

GÁbor Hamza, (Budapest), Ciceros Verhältnis zu seinen Quellen, mit besonderer Berücksichtigung der Darstellung der Staatslehre in De re publica . . . . Günther Christian Hansen, (Berlin), Das Datum der Schlacht bei Vercellae László Havas, (Debrecen), Geschichtsphilosophische Interpretationsmöglichkeiten bei Cornelius Nepos . . . . . . . . . . . . . . . . . . . . .

JoACHIm Henning, (Berlin), Ökonomie und Gesellschaft Rätiens zwischen Antike und Mittelalter . . . . . . . . . . . . . . . . . . . . . . . . . . . . .

Hans Joachim Herrmann, (Greifswald), Zur Bewertung und Interpretation antiker Historiker im 18. Jahrhundert in Deutschland . . . . . . . . .

Joachim Herrmann (Berlin), Gesellschaften im Untergang . . . . . . . . .

Friederike Heubner, (Jena), Agitatorische Redundanz als Mittel der politischen Argumentation in Ciceros Rede Pro Archia Poeta . . . . . . . . .

Walter HofmanN, (Leipzig), Über das antike Theater . . . . . . . . . . .

Tonio Hölscher, (Heidelberg), Denkmäler der Schlacht von Actium. Propaganda und Resonanz . . . . . . . . . . . . . . . . . . . . . . .

Liselot Huchthausen, (Rostock), Man darf auf die nächste Arbeit gespannt sein

Liselot Huchthausen, (Rostock), Spannende Lektüre . . . . . . . . . . 362

Liselot Huchthausen, (Rostock), Cicero und die Sklaverei. Zur Auswertung indirekter Aussagen . . . . . . . . . . . . . . . . . . . . . . . .

IASELOT HUCHTHAUSEN, (Rostock), Namen - vorwärts und rückläufig . . . . 608

IISELot Hбсhтна USEN, (Rostock), Kolonen - aber kein Kolonat! . . . . . . 618

Johan nes Irmscher, (Berlin), Vergil in der griechischen Antike . . . . . . . 281

Klaus-Peter Johne, (Berlin), Kaiser und Aristokratie des 3. bis 5. Jahrhunderts im Spiegel der prosopographischen Forschung . . . . . . . . . . . . . 337

Jana Kepartová, (Berlin), Ein Katalog der Antiken im Stadtbild von Chiusi $\quad$. 356

Jana Kepartová, (Berlin), Ein Katalog der Steininschriften von Concordia . . 358

Jana Kepartová, (Prag), Eine Einführung in die Epigraphik . . . . . . . . 609

Jana Kepartová, (Prag), Ehrenstatuen in Rom . . . . . . . . . . . . . . 612

JANa Kepartová, (Prag), Zu Inschriften aus Italien . . . . . . . . . . . . . . . 614

Jana Kepartová, (Prag), Ein Stellenregister . . . . . . . . . . . . . . . 617

Wolfgang KIrsch, (Halle/S.), Die Augusteische Zeit. Epochenbewußtsein und Epochenbegriff . . . . . . . . . . . . . . . . . . . 43

Ernst Kluwe, (Jena), Bildnispropaganda und römischer Kunsthandel . . . . . 103

HeLga Köpstein, (Berlin), Zum Versuch einer neuen Sicht auf die frühe byzantinische Entwicklung . . . . . . . . . . . . . . . . . . . . . . . . 633

REINhard KoERner, (Berlin), Tiryns als Beispiel einer friihen dorischen Polis . 452

Rennhard Koerner, (Berlin), Studien zum Attischen Seebund . . . . . . . . 592

Reinhard Koerner, (Berlin), Zur Gewaltenteilung im Athen des 4. Jh. v. u. Z. . 595

ReINHaRD Koerner, (Berlin), Diebstahl im attischen Recht . . . . . . . . . 597

ReinhaRd KoERner, (Berlin), Beiträge zu Einzelfragen der alten Geschichte . 601

Józef Korpanty, (Kraków), Furor in der Augusteischen Literatur .... . . 248

HeInz Kreissig $\dagger$, (Berlin), Weiteres zum Hellenismus . . . . . . . . . . . 603

Friedmar KüHnert, (Jena), Augusteische Dichtung und Dichtung der Augusteischen Zeit . . . . . . . . . . . . . . . . . . . . . . . . . . . 118

Hansulrich Labuske, (Berlin), Viel Streit um Homer . . . . . . . . . . 375 
Detref Lotze, (Jena), Märchenprinz und Tyrann : der Erbe Caesars . . . . . Helmut MaAss, (Berlin), Bemerkungen zur Rolle der Volksmassen in der Zerfalls- und Untergangsphase des Weströmischen Reiches ． . . . . . . 536

Helmut MaAss, (Berlin), Korporationen kontra Kaiser? . . . . . . . . . . 623

Józef Mantke, (Wroclaw), Aeneis als Epostitel . . . . . . . . . . . . . . 238

Stanislaw Mrozek, (Gdańsk), Die gesellschaftliche Rolle der Arbeit in der Augusteischen Zeit . . . . . . . . . . . . . . . . . . . . . . . 65

Remmar Müller, (Berlin), Prinzipatsideologie und Philosophie bei Horaz . . . 158

Manfred OpPermann, (Halle/S.), Der Ostbalkanraum in Augusteischer Zeit . . 111

Mari Pitschchadse, (Tbilissi), Prometheus in der römischen Poesie der Augusteischen Zeit . . . . . . . . . . . . . . . . . . . . . . . . . . . .

Elemér PólaY, (Debrecen), Historische Interpretationen der Generalklauseln im römischen Recht . . . . . . . . . . . . . . . . . . . . . 528

ILse Rochow, (Berlin), Zwei Neuerscheinungen zur Chronik des Theophanes . . 646

AnNa SADURSkA, (Warschau), L'art d'Auguste: progrès, regrès, ou transformation . . . . . . . . . . . . . . . . . . . . . .

JIMITRIos SAMSARIS, (Thessaloniki), Une inscription latine inédite trouvée près des frontières du territoire de la colonie romaine de Philippes . . . . .

Ernst A. Schmidt (Tübingen), Geschichtlicher Bewußtseinswandel in der Horazischen Lyrik . . . . . . . . . . . . . . . . . . . . . . . . . . .

ERnst Günther Schmidt, (Jena), Der politische, der unpolitische und der ganze

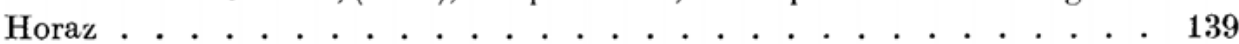

Edith Schönert-Geiss, (Berlin), Zur Existenz der pontischen Münzliga . . . 466

Thomas Sternberg, (Rostock), Reskripte des Kaisers Alexander Severus an weibliche Adressaten

Gotthard StrohmaIer, (Berlin), Aristoteles' De caelo inı „Großen Kommentar“ des Averroes . . . . . . . . . . . . . . . . . . . . . . . . . . .

Hanna Szelest, (Warschau), Die Augusteischen Dichter in den Adnotationes super Lucanum . . . . . . . . . . . . . . . . . . . . . . . . . .

Winfried Trillitzsch, (Jena), Augusteische Dichtung im deutschen Renaissancehumanismus. Das dichterische Werk des Erzhumanisten Conrad Celtis

Ilse UlmanN, (Berlin), Ergebnisse der neueren Forschung zu Ammianus Marcellinus . . . . . . . . . . . . . . . . .

Akakt URUSchadse, (Tbilissi), Vergils vierte Ekloge . . . . . . . . . . . . Andrew Wallace-Hadrill, (Cambridge), Progaganda and Dissent? Augustean Moral Legislation and the Love-Poets . . . . . . . . . . . . . . . .

Friedhelm Winkelmann, (Berlin), Ivar August Heikels Korrespondenz mit Hermann Diels, Adolf Harnack und Ulrich von Wilamowitz-Moellendorf . . Antonte Wrosok, (Mainz), Zur Funktion des Helden (Aeneas) in Vergils Aeneis . Genrikas Zabulis, (Vilnius), Die neue Auffassung von der Menschenwürde in Ovids Ars armatoria . . . . . . . . . . . . . . . . . . . . . . . . Gerhard Zinserling, (Jena), Die Programmatik der Kunstpolitik des August us $20 \check{5}$

Bis zum 15. 12. 1984 bei der Redaktion der Klio eingesandte Literatur . . . . 658 
